\title{
The plasticity and potential of leukemia cell lines to differentiate into dendritic cells (Review)
}

\author{
QINGWEI GUO ${ }^{1}$, LELING ZHANG $^{1}$, FU LI $^{1}$ and GUOSHENG JIANG ${ }^{2}$ \\ ${ }^{1}$ Qilu Children's Hospital of Shandong University, Jinan 250022; ${ }^{2}$ Institute of Basic Medicine, \\ Shandong Academy of Medical Science, Shandong Key Laboratory for Rare and Uncommon Diseases, \\ Jinan 250062, P.R. China
}

Received April 11, 2012; Accepted July 10, 2012

DOI: $10.3892 / \mathrm{ol} .2012 .821$

\begin{abstract}
Dendritic cells (DCs) are potent antigen-presenting cells that orchestrate the innate and adaptive immune systems to induce immunity. DCs are significant in maintaining immune tolerance towards self-antigens, organ transplantation and allergic responses. DCs are powerful adjuvants for eliciting T-cell immunity and are therefore considered primary targets for inducing immune responses in the prevention and treatment of infectious diseases and cancer. DCs have been increasingly applied in the immunotherapy of cancer worldwide during the last decade; however, a number of the highly specialized biological characteristics of DCs remain to be elucidated. Previous studies of human DCs have been constrained by certain difficulties, therefore the majority of studies have been carried out using in vitro model systems. Suitable cell lines with dendritic-like properties may provide valuable tools for the study of DC physiology and pathology. In the current review, various human DC line differentiation models are discussed. Certain cell lines provide valuable tools for studying the specific aspects of DC biology, despite variations in cell biological and immunological features when compared with primary DCs.
\end{abstract}

\section{Contents}

1. Introduction

2. HL-60 cells

3. NB4 cells

4. MUTZ-3 cells

5. THP-1 cells

6. K562 cells

7. Concluding remarks

Correspondence to: $\mathrm{Dr}$ Guosheng Jiang, Institute of Basic Medicine, Shandong Academy of Medical Science, Shandong Key Laboratory for Rare and Uncommon Diseases, 23976 Jingshi Road, Shandong, Jinan 250062, P.R. China

E-mail: jiangguosh@hotmail.com

Key words: dendritic cells, differentiation models, valuable tools

\section{Introduction}

Dendritic cells (DCs) are hematopoietic cells that belong to the antigen-presenting cell (APC) family, which also includes $\mathrm{B}$ cells and macrophages. They are responsible for the induction of cellular immune responses, by recognizing, acquiring, processing and presenting antigens to naïve, resting $\mathrm{T}$ cells for the induction of an antigen-specific immune response. Of all the antigen presenting cells, DCs are the most effective inducers of T-cell-based immunity (1). DCs originate from myeloid precursors that, during their development, progress from the blood into the peripheral tissues. DCs reside in the peripheral tissues in an immature state (iDCs) equipped with specialized receptors, including toll-like receptors, C-type lectins, the cytoplasmic NOD/NALP family, RIG-I/DDX58 and MDA5/IFIH1 molecules and Fc receptors, where they capture and process antigens for presentation in the context of MHC molecules (2). iDCs undergo a maturation process in response to pathogens, antigens and/or pro-inflammatory signals, during which they acquire the morphological, phenotypical and functional characteristics of mature DCs (mDCs). During maturation, DCs upregulate the major histocompatibility complex (MHC) and remodel their surfaces, typically expressing numerous membrane-associated co-stimulatory molecules (including members of the B7, TNF and notch families) (3). Subsequently, mDCs progress into the secondary lymphoid tissues, where they present the processed antigens to naïve $\mathrm{T}$ cells to generate effector T cells. Since DCs are so well equipped for initiating adaptive immune responses, they are considered to be prime targets for modulating immune responses against cancer. In the overt absence of maturation stimuli, steady state DCs induce tolerance when they capture self and environmental antigens (4).

Worldwide, research is being conducted to explore the therapeutic application of DCs; however, numerous aspects of DC-biology remain poorly understood. Studies of human DCs have been constrained by various factors. Firstly, differentiated DCs are predominantly located in the tissue rather than the blood, making isolation and analysis more difficult. Although DCs are isolated from either the spleen (5) or blood $(6,7)$, their low and varying levels often hamper studies by making it difficult to generate large amounts of immunostimulatory DCs. Second, the life span of the culture 
is limited since DCs are terminally differentiated and cannot divide. Although larger numbers of DCs may be derived by the in vitro differentiation of bone marrow stem cells (8), peripheral blood mononuclear cells (9) or monocytes (10), the isolation of these DC-precursors is laborintensive and the generation of typical DCs is dependent on the differentiative stage of the primary culture. The subsequent differentiation is also time consuming. Third, these cultures do not sustain DC production for long periods of time and do not allow the identification or study of the intermediate stages as the majority of cytokine-supplemented cultures drive progenitors quickly to mDCs. Moreover, the current methods of preparation $e x$ vivo or from primary cell culture are not only laborious, but suffer from the inherent difficulties of reproducibility associated with the study of primary human material. Finally, experiments using these primary cells are often complicated by donor-donor variations in cytokine expression levels, cell surface molecule expression levels and the ability to stimulate $\mathrm{T}$ cells. Hence, there is a significant requirement for model cell lines to aid in the understanding of the developmental origins of DCs and the aspects that are induced to undergo maturation in response to pathogens or cytokine stimulation. It has been described that the 'dendritic-like' cells are established from the leukemia-derived cell lines that are capable of differentiating into functional DCs (37). This creates possibilities for the development of highly reproducible DCs and providing in vitro model systems for in-depth studies concerning DC physiology.

This review outlines a number of human DC line differentiation models, various leukemia cell lines, multiple differentiation potentials and the plasticity to differentiate into DCs. The evidence suggests that various leukemia cell lines retain the potential for terminal differentiation into diverse peripheral mature cell types, even DCs.

\section{HL-60 cells}

The HL-60 cell line, derived from a patient with acute promyelocytic leukemia (APL), may be induced in vitro to differentiate into numerous cell types. Studies using this leukemic cell line have been invaluable in a variety of areas. HL-60 is induced to differentiate into granulocytelike cells by incubation with a wide variety of compounds, including DMSO and retinoic acid $(11,12)$ or into monocyte (macrophage)-like cells by incubation with 1,25-dihydroxyvitamin D3 (VD3) or phorbol esters (13-15). Moreover, the HL-60 cell line is also capable of differentiating into eosinophilic granulocytes when cultured under mildly alkaline conditions (16). The HL-60 cell line expresses MHC class I molecules (17), but lacks the expression of MHC class II and costimulatory molecules (18). The demonstrated capacity to differentiate in vitro into cells exhibiting numerous characteristics of various myeloid-lineage cell types proposed the sensitivity of the HL-60 cell line for differentiation along the myeloid DC pathway, whereas studies using cytokines to promote the DC differentiation of the HL-60 cell line were not successful $(19,20)$. Treatment with cytokines did not induce the enhanced expression of costimulatory nor MHC molecules. Inclusion of calcium ionophore (CI) mobilization treatment resulted in a more mature phenotype: $\mathrm{CI}$ induced the HL-60 cell line to upregulate CD83 and CD86 expression and to acquire dendritic processes, characteristics that are associated with the mature, activated DC phenotype. Of note, $\mathrm{CI}$ treatment also resulted in a marked increase in APC function, as determined by enhanced allogeneic T-cell stimulation capacity. However, the T-cell stimulatory capacity was low, as CI treatment failed to induce MHC class II molecules and downregulate MHC class I molecules. HL-60 cells only upregulated the expression of MHC class II molecules when induced with a combination of ionophore and inteferon- $\gamma$. The mechanism by which CI induced the differentiation of HL-60 cells into DCs was related to triggering a downstream signal transduction pathway. Protein kinase C (PKC) plays a role in determining the capacity of $\mathrm{CI}$ to induce leukemic cell differentiation and the blockade of PKC with bisindolylmaleimide-I (Bis-1) inhibited the differentiation of HL-60 myeloblasts into leukemic DCs with CI $(21,22)$.

\section{NB4 cells}

The NB4 cell line, a human promyelocytic leukemia cell line, is the only permanent cell line with $\mathrm{t}(15 ; 17)$ established from the leukemic cells of a patient with APL. The $t(15 ; 17)$ translocation produces a chimeric protein called PML-RAR $\alpha$ (23). NB4 cells were instrumental in the molecular characterization of the PML-RAR $\alpha$ fusion gene (24). Although HL-60 was previously known as 'promyelocytic', it has since been revealed that it was derived from an acute promyelocytic leukemia with maturation (M2). Moreover, it does not contain the typical $\mathrm{t}(15 ; 17)$ translocation (25). NB4 cells undergo differentiation via the granulocytic pathway when exposed to all-trans retinoic acid (ATRA) (23). ATRA mediates its effect via specific nuclear retinoid receptors (26). The further differentiation of leukemic promyelocytes into DC-like cells following their differentiation into granulocytes by ATRA has also been studied (24). The differentiation of NB4 cells by ATRA causes the cells to express DC markers that enable ATRA-differentiated NB4 cells to present antigens to, and hence activate, T cells. NB4 cells upregulate the markers identified in DCs, including HLA-DR, costimulatory molecules (CD80 and CD86), adhesion molecules (CD40) and chemokine receptors (CCR6) in the presence of ATRA. High levels of expression of CD83, a specific surface marker of DC maturation (27), were also detected on the surface of ATRA-treated NB4 cells. The mechanism by which ATRA-differentiated NB4 cells are induced into DC-like cells involves the NF- $\mathrm{KB}$ pathway $(28,29)$. Previous research has demonstrated that phosphatidic acid (PA) also differentiates the NB4 cells into DC-like cells (30). The expression of DC markers, including MHC-II, CD11c, CD80 CD86 and CD83, was reported to be upregulated in PA-treated NB4 cells (30). Increased functional capacities were also revealed in PA-differentiated NB4 cells with regard to changes in T-cell proliferation, cytokine production, endocytic activity and cytolytic capacity. The downregulation of PML-RAR $\alpha$ or related signaling pathways, including ERK, may mediate the differentiation signals in the NB4 cells exposed to PA. However, further research is required to identify the downstream target of ERK-1/2 that is involved in the PA-induced differentiation of NB4 cells into DC-like cells. 


\section{MUTZ-3 cells}

The human acute myelomonocytic leukemic cell line MUTZ-3 may provide an alternative model for DC studies. Several previous publications have proposed that a proportion of MUTZ-3 cells exhibit the phenotypic and morphological characteristics that resemble those of DCs and that these cells undergo further maturation and acquire the ability to activate resting $\mathrm{T}$ cells $(31,32)$.

MUTZ-3 is a human myeloid leukemia cell line established from a 29-year-old male, which carries the inv(3)(q21q26) and the $\mathrm{t}(12 ; 22)$ (p13;ql1) chromosomal rearrangements (33). It exhibits the morphological and phenotypical characteristics of monocytes, as suggested by its expression of monocyte-specific esterase and myeloperoxidase enzymes and the expression of the monocytic marker CD14. The MUTZ-3 cell line consists of three distinct subpopulations, a proliferating pool of small

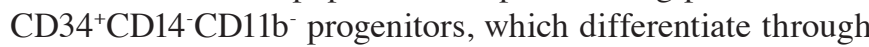
an intermediate $\mathrm{CD} 34^{-} \mathrm{CD} 14^{-} \mathrm{CD} 11 \mathrm{~b}^{+}$stage to ultimately give rise to a morphologically large, more differentiated, nonproliferating $\mathrm{CD} 14^{+} \mathrm{CD} 11 \mathrm{~b}^{\mathrm{hi}}$ precursor population during cytokine-dependent culture. This maintained capacity has been described to be cytokine-dependent for its proliferation and survival (33). Masterson et al have demonstrated that MUTZ-3 cells have the potential to differentiate into Langerhans-like cells upon the addition of a cocktail of differentiating cytokines (34). Over the course of MUTZ-3 differentiation, cytokine receptors that are associated with DC differentiation, including GM-CSF-R and TNF-R, are upregulated (35). The MUTZ-3 cell line has been shown to downregulate CD14 in response to GM-CSF and IL-4 and to exhibit the characteristics of CD34-derived DC precursors (34), showing a unique potential for the phenotypical differentiation into functional DCs with discrete immature and mature stages. Culturing MUTZ-3 cells in GM-CSF, IL-4 resulted in proliferation arrest, cell differentiation and the neoexpression of CDla. The culture of MUTZ-3 iDCs with high TNF $\alpha$ induced the neoexpression of the DC maturation marker CD83 with further upregulation of CD1a. MUTZ-3-derived DC (MuDC) maturation was also induced by CD40-mediated stimulation and demonstrated a high level of expression of HLA class II molecules, CD80 and CD86 (36). It has been further demonstrated that MuDCs possess the capacity to acquire a functional cytokine-induced DC phenotype, which exhibits the full range of functional antigen-processing and presentation pathways and exhibits functional properties that are essential for the in vivo generation of cytotoxic T lymphocyte (CTL)-mediated immunity $(37,38)$. Signals that promote DC maturation and survival may follow various pathways. It has been shown that the maturation of DCs is mediated by the activation of $\mathrm{p} 38$-mitogen-activated protein kinase (MAPK) (39).

Furthermore, on the basis of the comparative functional and transcriptional profiles of MuDCs and monocyte-derived DCs used as a standard source of DCs, MUTZ-3 exhibited a gene induction similar to that of monocyte-derived DCs (31).

The ability to differentiate into DCs has led scholars to investigate the MUTZ-3 cell line as a potential in vitro model for the identification of skin allergens $(40,41)$. Indeed, the immortalized human MUTZ-3 cell line constitutes an unlimited supply of DC precursors and is a potentially useful tool for the generation of stable transfectants for the further elucidation of DC differentiation pathways.

\section{THP-1 cells}

THP-1 is a human monocytic leukemia cell line that was cultured from the blood of a 1-year-old male with acute monocytic leukemia (42). THP-1 has been used not only as a clinical model of a leukemic cell, but also as a scientific model of differentiation in response to various stimuli. On stimulation with phorbol 12-myristate 13-acetate (PMA) or VD3, which activates PKC, THP-1 cells cease proliferation, become adherent and differentiate into macrophage-like cells (43). IL-32 also induces the differentiation of the THP-1 cells into macrophage-like cells, the expression of CDla, a DC marker, and amplifies the effects of GM-CSF/IL-4 on CD83 expression. These observations demonstrate that IL-32 induces the differentiation of monocytes into a phenotype that exhibits an increase in certain DC markers. The effect of IL-32 on monocyte differentiation appears to be dependent on caspase-3 activity (44). Moreover, previous studies have identified that the THP-1 cell line is differentiated rapidly into mature DCs when cultured in serum-free medium containing GM-CSF, TNF- $\alpha$ and ionomycin (45).

Generally, only the use of a serum-free medium complemented with GM-CSF and TNF- $\alpha$ results in the differentiation into iDCs. The culturing of THP-1 cells in a serum-free medium supplemented with ionomycin resulted in a complete differentiation of the cells into mDCs (45). These THP-1 cell line-derived highly pure DCs exhibit the morphological, phenotypical, molecular and functional properties, including characteristic DC morphology and cell-surface molecule expression profiles, as determined by the cell-surface expression of CD83, CD80, CD86, CD40, CD206, CD209, CD120 $(46,47)$, endocytotic activity $(48,49)$ and strong T-cell stimulatory capacity $(50,51)$. Ionomycin is a CI that mediates the intracellular calcium flux by increasing the cell membrane permeability to $\mathrm{Ca}^{2+}$. Mobilization of intracellular calcium has been shown to activate the signaling pathways known to be induced in response to cytokine stimulation and finally converges on the activation and nuclear translocation of transcription factors of the NF-jB/Rel family, leading to DC maturation $(52,53)$.

\section{K562 cells}

The K562 cell line was originally established from a pleural effusion of a patient with chronic myelogenous leukemia in terminal blast crisis (54), which exhibits the Philadelphia (Ph) chromosome, an aberration involving a 9:22 chromosomal translocation identified in $>90 \%$ of chronic myelogenous leukemia cases (55). These cells exhibit erythroid, granulocytic, monocytic or megakaryocytic markers (56), which suggests that they may result from the transformation of a multipotential hematopoietic precursor. K562 cells have been extensively used as an in vitro model system for studying the differentiation along the erythroid lineage. Following treatment with hemin, 5-azacytidine, 1-/3-D-arabinofuranosylcytosine, daunomycin or erbimycin, the cell line is induced to differentiate into an erythroid lineage (57-59). By contrast, 


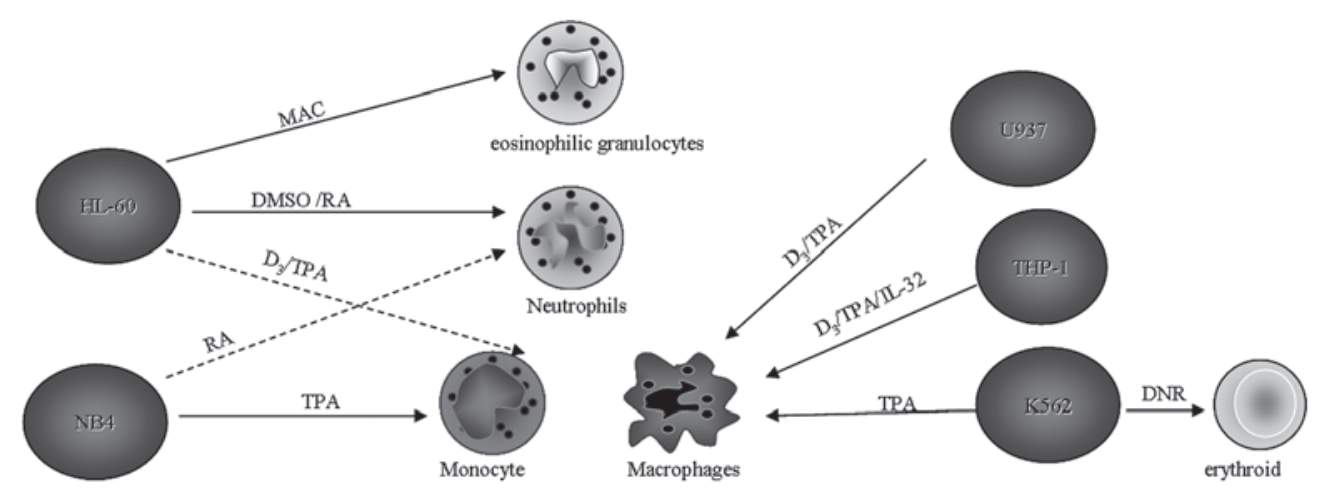

Figure 1. Schematic diagram illustrating trans-lineage differentiation of HL-60, NB4, U937, THP-1 and K562 cells. MAC, mild alkaline conditions; RA, retinoic acid; DMSO, dimethyl sulfoxide; TPA, 12-O-tetradecanoylphorbol-13-acetate; DNR, daunomycin.

phorbol dibutyrate and phorbol 12-myristate 13-acetate (TPA) induce differentiation into monocytes (60).

In addition, upon stimulation with PMA plus TNF, K562 cells develop DC-like cytoplasmic projections, but the expression of typical DC markers, including CD86, CD40 and CD83, remains low. However, PMA plus A23187 was found to induce differentiation into cells with typical DC morphology, characteristic surface markers (MHC class I, MHC class II, CD86, CD40, CD83), chemokine and transcription factor expression and the ability to stimulate T-cell proliferation. The mechanisms underlying this differentiation process concerned with the downregulation of BCR-ABL gene expression specifically involve downstream signaling through the MAPK pathway (61).

\section{Concluding remarks}

DCs play a fundamental role in modulating the innate and adaptive immune responses. Thus, they have evoked considerable interest as potential tools for the development of cell therapies to induce antitumor immune responses. There has therefore been enormous interest in understanding the function of these cells and in applying this to immunotherapy. DCs may be isolated as terminally differentiated, post-mitotic cells from all tissues and in the blood, but they comprise only a small proportion (less than $0.1 \%$ of circulating leukocytes). DCs may also be obtained from adherent peripheral blood monocytes and $\mathrm{CD}_{3} 4^{+}$stem cells in vitro. The relatively low numbers of cells that may thus be obtained, compared with the difficulties: laborintensity and reproducibility associated with the study of primary human material in vitro and in vivo, have seriously hampered studies aimed at exploring the cell biology of DCs. Studies of human DCs have been constrained by such difficulties, therefore the majority of studies have been carried out using in vitro model systems. These model systems have proved extremely powerful, generating a large body of fundamental research describing numerous aspects of DC function, alongside a body of translational research using DCs for the cellular adoptive immunotherapy of infection and cancer.

A number of cell lines exhibiting the characteristics of DCs would allow for the detailed study of DC differentiation without the associated problems. Leukemic cell lines retain a degree of lineage plasticity and a number differentiate further in response to defined stimuli. Moreover, trans-lineage differentiation among erythroid, myeloid, megakaryocytic and lymphoid compartments have been also reported in vitro in the presence of selected cytokines or chemicals (Fig. 1) (62). This suggests that myeloid leukemias retain the potential for terminal differentiation into various peripheral mature cell types. Therefore, the flexible ability allows the leukemic cells to share a potential for differentiation into DCs.

The potential to differentiate into DCs has already been studied for several cell lines. Using diverse combinations of cytokines and hematopoietic differentiation agents, various human hematopoietic cell lines have the ability to differentiate into iDCs and mDCs with the phenotypic, molecular and functional properties of DCs. These model systems have proven extremely powerful, generating a large body of fundamental research describing numerous aspects of DC function, alongside a body of translational research using DCs for the cellular adoptive immunotherapy of infection and cancer.

Leukemia cell lines were predicted to be good candidates for differentiation into DCs, although MUTZ-3 is unique in comparison with other myeloid leukemic cell lines $(45,63)$ in that it allows the study of discrete transitional stages in myeloid DC development. No single cell line is likely to provide a perfect model for a normal cell phenotype. In spite of their limitations, a number of DC model cell lines may be successfully used to study significant aspects of DC biology in vitro. The MUTZ-3 cell line thus represents a valuable and sustainable model system for the further elucidation of the molecular mechanisms regulating the early and late stages of human myeloid DC differentiation.

We expect that the proper use of DC model cell lines may aid in the revelation of significant new insights into DC development, maturation, physiology and pathology that may subsequently be applied to improve the application of DCs in the clinic.

\section{References}

1. Banchereau J and Steinman RM: Dendritic cells and the control of immunity. Nature 392: 245-252, 1998.

2. Banchereau J, Briere F, Caux C, et al: Immunobiology of dendritic cells. Annu Rev Immunol 18: 767-811, 2000.

3. Spörri R and Reise e Sousa C: Inflammatory mediators are insufficient for full dendritic cell activation and promote expansion of CD4+ T cell populations lacking helper function. Nat Immunol 6: $163-170,2005$. 
4. Hawiger D, Inaba K, Dorsett Y, et al: Dendritic cells induce peripheral $\mathrm{T}$ cell unresponsiveness under steady state conditions in vivo. J Exp Med 194: 769-779, 2001.

5. Steinman RM and Cohn ZA: Identification of a novel cell type in peripheral lymphoid organs of mice. I. Morphology, quantitation, tissue distribution. J Exp Med 137: 1142-1162, 1973.

6. Van Voorhis WC, Hair LS, Steinman RM and Kaplan G: Human dendritic cells. Enrichment and characterization from peripheral blood. J Exp Med 155: 1172-1187, 1982.

7. Crow MK and Kunkel HG: Human dendritic cells: major stimulators of the autologous and allogeneic mixed leucocyte reactions. Clin Exp Immunol 49: 338-346, 1982.

8. Inaba K, Inaba M, Romani N, et al: Generation of large numbers of dendritic cells from mouse bone marrow cultures supplemented with granulocyte/macrophage colony-stimulating factor. J Exp Med 176: 1693-1702, 1992.

9. Romani N, Gruner S, Brang D, et al: Proliferating dendritic cell progenitors in human blood. J Exp Med 180: 83-93, 1994.

10. Kiertscher SM and Roth MD: Human CD14+ leukocytes acquire the phenotype and function of antigen-presenting dendritic cells when cultured in GM-CSF and IL-4. J Leukoc Biol 59: 208-218, 1996.

11. Collins SJ, Ruscetti FW, Gallagher RE and Gallo RC: Norma functional characteristics of cultured human promyelocytic leukemia cells (HL-60) after induction of differentiation by dimethylsulfoxide. J Exp Med 149: 969-974, 1979.

12. Breitman TR, Selonick SE and Collins SJ: Induction of differentiation of the human promyelocytic leukemia cell line (HL-60) by retinoic acid. Proc Natl Acad Sci USA 77: 2936-2940, 1980.

13. Miyaura C, Abe E, Kuribayashi T, et al: 1,25-Dihydroxyvitamin D3 induces differentiation of human myeloid leukemia cells. Biochem Biophys Res Commun 102: 937-943, 1981.

14. Ball ED, Guyre PM, Shen L, et al: Interferon induces monocytoid differentiation in the HL-60 cell line. J Clin Invest 73: 1072-1077, 1984.

15. Rovera G, Santoli D and Damsky C: Human promyelocytic leukemia cells in culture differentiate into macrophage-like cells when treated with a phorbol diester. Proc Natl Acad Sci USA 76: 2779-2783, 1979

16. Fischkoff SA, Pollak A, Gleich GJ, et al: Eosinophilic differentiation of the human promyelocytic leukemia cell line, HL-60. J Exp Med 160: 179-196, 1984.

17. Chorváth B, Sedlák J and Fuchsberger N: Interferon $\alpha$-induced modulation of leukocyte cell surface antigens: immunocytofluorometric study with human leukaemia/lymphoma cell lines. Acta Virol 35: 7-18, 1991.

18. Yunis JJ, Band H, Bonneville F and Yunis EJ: Differential expression of MHC class II antigens in myelomonocytic leukemia cell lines. Blood 73: 931-937, 1989.

19. Santiago-Schwarz F, Coppock DL, Hindenburg AA and Kern J: Identification of a malignant counterpart of the monocyte-dendritic cell progenitor in an acute myeloid leukemia Blood 84: 3054-3062, 1994

20. Koski GK, Schwartz GN, Weng DE, et al: Calcium ionophore-treated myeloid cells acquire many dendritic cell characteristics independent of prior differentiation state, transformation status, or sensitivity to biologic agents. Blood 94 1359-1371, 1999.

21. Li Q, Ozer H, Lindner I, et al: Protein kinase C blockade inhibits differentiation of myeloid blasts into dendritic cells by calcium ionophore A23187. Int J Hematol 81: 131-137, 2005.

22. Nguyen TN, Choi BH, Kang HK, et al: Optimization and limitation of calcium ionophore to generate DCs from acute myeloid leukemic cells. Cancer Res Treat 39: 175-180, 2007.

23. Lanotte M, Martin TV, Najman S, et al: NB4, a maturation inducible cell line with $\mathrm{t}(15 ; 17)$ marker isolated from a human acute promyelocytic leukemia (M3). Blood 77: 1080-1086, 1991.

24. Park HY, Park JY, Kim JW, et al: Differential expression of dendritic cell markers by all-trans retinoic acid on human acute promyelocytic leukemic cell line. Int Immunopharmacol 4 $1587-1601,2004$

25. Dalton WT, Ahearn MJ, McCredie KB, et al: HMO cell line was derived from a patient with FAB-M2 and not FAB-M3. Blood 71: 242-247, 1988

26. Pitha-Rowe I, Petty WJ, Kitareewan S and Dmitrovsky E: Retinoid target genes in acute promyelocytic leukemia. Leukemia 17: 1723-1730, 2003.

27. Zhou LJ and Tedder TF: Human blood dendritic cells selectively express CD83, a member of the immunoglobulin superfamily. J Immunol 154: 3821-3835, 1995.
28. Yoshimura S, Bondeson J, Foxwell BM, et al: Effective antigen presentation by dendritic cells is NF- $\mathrm{KB}$ dependent: coordinate regulation of $\mathrm{MHC}$, costimulatory molecules and cytokines. Int Immunol 13: 675-683, 2001

29. Berchtold S, Mühl-Zürbes P, Maczek E, et al: Cloning and characterization of the promoter region of the human CD83 gene. Immunobiology 205: 231-246, 2002.

30. Jin JO, Park HY, Kim JW, et al: Phosphatidic acid induces the differentiation of human acute promyelocytic leukemic cells into dendritic cell-like. J Cell Biochem 100: 191-203 2007.

31. Larsson K, Lindstedt $M$ and Borrebaeck CA: Functional and transcriptional profiling of MUTZ-3, a myeloid cell line acting as a model for dendritic cells. Immunology 117: 156-166, 2006.

32. Santegoets SJ, Schreurs MW, Masterson AJ, et al: In vitro priming of tumor-specific cytotoxic $\mathrm{T}$ lymphocytes using allogeneic dendritic cells derived from the human MUTZ-3 cell line. Cancer Immunol Immunother 55: 1480-1490, 2006.

33. Hu ZB, Ma W, Zaborski M, et al: Establishment and characterization of two novel cytokine responsive acute myeloid and monocytic leukemia cell lines, MUTZ-2 and MUTZ-3. Leukemia 10: $1025-1040,1996$

34. Masterson AJ, Sombroek CC, De Gruijl TD, et al: MUTZ-3, a human cell line model for the cytokine induced differentiation of dendritic cells from CD34+ precursors. Blood 100: 701-703, 2002.

35. Santegoets SJ, Masterson AJ, van der Sluis PC, et al: A CD34(+) human cell line model of myeloid dendritic cell differentiation: evidence for a CD14(+)CD11b(+) Langerhans cell precursor. J Leukoc Biol 80: 1337-1344, 2006.

36. Kim KD, Choi SC, Noh YW, et al: Impaired responses of leukemic dendritic cells derived from a human myeloid cell line to LPS stimulation. Exp Mol Med 38: 72-84, 2006.

37. Santegoets SJ, van den Eertwegh AJ, van de Loosdrecht AA et al: Human dendritic cell line models for DC differentiation and clinical DC vaccination studies. J Leukoc Biol 84: 1364 $1373,2008$.

38. Nelissen I, Selderslaghs I, Heuvel RV, et al: MUTZ-3-derived dendritic cells as an in vitro alternative model to CD34+ progenitor-derived dendritic cells for testing of chemical sensitizers. Toxicol In Vitro 23: 1477-1481, 2009.

39. Arrighi JF, Rebsamen M, Rousset F, et al: A critical role for $\mathrm{p} 38$ mitogen-activated protein kinase in the maturation of human blood-derived dendritic cells induced by lipopolysaccharide, TNF- $\alpha$ and contact sensitizers. J Immunol 166: 3837-3845, 2001.

40. Hulette BC, Ryan CA and Gerberick GF: Elucidating changes in surface marker expression of dendritic cells following chemical allergen treatment. Toxicol Appl Pharmacol 182: 226-233, 2002.

41. Azam P, Peiffer JL, Chamousset D, et al: The cytokine-dependent MUTZ-3 cell line as an in vitro model for the screening of contact sensitizers. Toxicol Appl Pharmacol 212: 14-23, 2006.

42. Tsuchiya S, Yamabe M, Yamaguchi Y, et al: Establishment and characterization of a human acute monocytic leukemia cell line (THP-1). Int J Cancer 26: 171-176, 1980.

43. Schwende H, Fitzke E, Ambs P and Dieter P: Differences in the state of differentiation of THP-1 cells induced by phorbol ester and 1,25-dihydroxyvitamin D3. J Leukoc Biol 59: 555-561, 1996

44. Netea MG, Lewis EC, Azam T, et al: Interleukin-32 induces the differentiation of monocytes into macrophage-like cells. Proc Natl Acad Sci USA 105: 3515-3520, 2008

45. Berges C, Naujokat C, Tinapp S, et al: A cell line model for the differentiation of human dendritic cells. Biochem Biophys Res Commun 333: 896-907, 2005.

46. Rosenzwajg M, Canque B and Gluckman JC: Human dendritic cell differentiation pathway from CD34+ hematopoietic precursor cells. Blood 87: 535-544, 1996.

47. Zhou LJ and Tedder TF: CD14+ blood monocytes can differentiate into functionally mature CD83+ dendritic cells. Proc Natl Acad Sci USA 93: 2588-2592, 1996.

48. Sallusto F, Cella M, Danieli C and Lanzavecchia A: Dendritic cells use macropinocytosis and the mannose receptor to concentrate macromolecules in the major histocompatibility complex class II compartment: downregulation by cytokines and bacterial products. J Exp Med 182: 389-400, 1995.

49. Monti P, Mercalli A, Leone BE, et al: Rapamycin impairs antigen uptake of human dendritic cells. Transplantation 75: 137-145, 2003.

50. Sallusto F and Lanzavecchia A: Efficient presentation of soluble antigen by cultured human dendritic cells is maintained by granulocyte/macrophage colony-stimulating factor plus interleukin 4 and downregulated by tumor necrosis factor $\alpha$. J Exp Med 179: $1109-1118,1994$. 
51. Ferlazzo G, Wesa A, Wei WZ and Galy A: Dendritic cells generated either from CD34+ progenitor cells or from monocytes differ in their ability to activate antigen-specific CD8+ T cells. J Immunol 163: 3597-3604, 1999.

52. Lyakh LA, Koski GK, Telford W, et al: Bacterial lipopolysaccharide, TNF- $\alpha$, and calcium ionophore under serum-free conditions promote rapid dendritic cell-like differentiation in CD14+ monocytes through distinct pathways that activate NK-jB. J Immunol 165: 3647-3655, 2000.

53. Faries MB, Bedrosian I, Xu S, et al: Calcium signaling inhibits interleukin-12 production and activates CD83+ dendritic cells that induce Th2 cell development. Blood 98: 2489-2497, 2001.

54. Lozzio CB and Lozzio BB: Human chronic myelogenous leukemia cell line with positive Philadelphia chromosome. Blood 45: 321-334, 1975.

55. Fialkow PJ: Clonal origin of human tumors: Annu Rev Med 30: 135-143, 1979.

56. Green AR, Rockman S, DeLuca E and Begley CG: Induced myeloid differentiation of K562 cells with downregulation of erythroid and megakaryocytic transcription factors: a novel experimental model for hemopoietic lineage restriction. Exp Hematol 21: 525-531, 1993.

57. Bianchi Scarrá GL, Romani M, Coviello DA, et al: Terminal erythroid differentiation in the K-562 cell line by 1-beta-Darabinofuranosylcytosine: accompaniment by c-myc messenger RNA decrease. Cancer Res 46: 6327-6332, 1986.
58. Tonini GP, Radzioch D, Gronberg A, et al: Erythroid differentiation and modulation of c-myc expression induced by antineoplastic drugs in the human leukemic cell line K562. Cancer Res 47: 4544-4547, 1987.

59. Honma Y, Okabe-Kado J, Hozumi M, et al: Induction of erythroid differentiation of K562 human leukemic cells by herbimycin A. an inhibitor of tyrosine kinase activity. Cancer Res 49: 331-334, 1989.

60. Sutherland JA, Turner AR, Mannoni P, et al: Differentiation of K562 leukemia cells along erythroid, macrophage, and megakaryocyte lineages. J Biol Response Mod 5: 250-262, 1986.

61. Lindner I, Kharfan-Dabaja MA, Ayala E, et al: Induced dendritic cell differentiation of chronic myeloid leukemia blasts is associated with down-regulation of BCR-ABL. J Immunol 171: 1780-1791, 2003.

62. Montanari M, Gemelli C, Tenedini E, et al: Correlation between differentiation plasticity and mRNA expression profiling of CD34+-derived CD14- and CD14+ human normal myeloid precursors. Cell Death Differ 12: 1588-1600, 2005.

63. Platzer B, Jörgl A, Taschner S, et al: RelB regulates human dendritic cell subset development by promoting monocyte intermediates. Blood 104: 3655-3663, 2004. 